\title{
Media Access Control Protocol in Ad Hoc Network based on Hybrid Antennas
}

\author{
Ming-ce CHENG, Ying LI \\ Department of computer and science, Soochow University, Suzhou, China
}

(20104227020, ingli)@suda.edu.cn

Keywords: Ad hoc network; DFMAC protocol; Hybrid antennas; Network simulator; Gain.

\begin{abstract}
In order to solve the problem of the communication asymmetry problems in hybrid ad hoc network because of the introduction of directional antennas, we propose a hybrid MAC protocol termed DFMAC protocol, which achieving data transmission by one hop through the use of exchange RTS by multiple hops. The protocol takes the benefit of directional antennas and can be used by node equipped with Omni-directional antennas, simulation results show that the DFMAC protocol performs well in improving the network throughput.
\end{abstract}

\section{Introduction}

Directional antenna technology provide many potential benefits for wireless communication systems, Using directional antennas has been proved that it is a good choice to improve the performance [1, 2]. Reference [3] was shown that directional antennas can gain $4 \pi 2 / \alpha \beta$ more capacity in random ad hoc networks, compared with Omni-antennas, where $\alpha$ and $\beta$ are the beamwidths of the transmitter and receiver.

However, in wireless ad hoc network, the nodes may be equipped with different antenna. Many MAC protocols which designed for only one kind of antennas are not suitable of such networks. For example, MAC protocols based on directional antenna usually use directional characteristics to estimate the DOA (direction of angle) of the signal, which often can not be directly applied to Omni-directional nodes. If use a MAC protocol based on Omni-directional antenna in wireless ad hoc network, it can not take advantage of the directional antenna.

Few solutions have been proposed in the past few years and the research about hybrid network just begin. K. Sundaresan and R. Sivakumar [4] studied architecture of hybrid network, including the principles of routing protocol and MAC protocol, but they did not give a specific and concrete implementation. M. Takai et al [5] proposed a MAC mechanisms called DVCS, this protocol can be used in simple hybrid network, when the transmitter does not know the DOA of the receiver, it will send an Omni-directional RTS (Ready To Send) to reserve the channel, the receiver will reply CTS (Clear To Send) to inform the transmitter that channel reservation is successful. However, the use of ORTS/OCTS make the protocol can only reach a distance of the O-O (Omni-Omni), failed to employ the advantage of the directional antenna. In [6], the authors assumed that the directional node has all capacity of directional and Omni-directional antenna, if one of the transmitter and the receiver is an Omni-directional nodes, it will use DCF model, and if both of them are equipped with directional antenna, the communication use DDCF (directional DCF) model. This protocol make the communication range between a normal node and directional node only reach $\mathrm{O}-\mathrm{O}$ distance, and when the data is transmitting, the protocol is unable to realized spatial reuse.

In order to employ benefits of directional antennas, in this paper, a protocol termed DFMAC based on the hybrid antenna is proposed. The protocol can realize one hop data transmission through exchanging multi-RTS in the network.

\section{Protocol Descriptions}

Assume that there are two kinds of nodes in wireless ad hoc network, each node is equipped with a transceiver, normal node (equipped with Omni-directional antenna) can finish Omni-directional 
transmitting and directional receiving, directional node can take directional transmitting and directional receiving, besides that, it will in Omni-directional mode when it is in idle state. The energy obtained from transmitter can be calculated by the TwoRayGround model (Eq. 1).

$$
P_{r}=G_{t} G_{r} \frac{h_{a}^{2}+h_{b}^{2}}{d_{a b}^{4}}
$$

Where $G_{t}, G_{r}$ respectively represents the gain of the transmitter and receiver, $h_{a}, h_{b}$ represents the node height respectively, $\mathrm{d}_{\mathrm{ab}}$ represents the distance of node $\mathrm{a}$ and node $\mathrm{b}$.

Assuming the gain of Omni-directional antenna and directional antenna are Go and $\mathrm{Gm}$ (Gm> Go). When a normal node has data to send to directional node which in listening mode, the gain of the transmitter(Gt) and receiver(Gr) is Go; while a directional node transmits to a normal node, the gain $\mathrm{Gt}=\mathrm{Gm}$ and $\mathrm{Gr}=\mathrm{Go}$. Therefore, the communication distance of a normal node transmits to a directional node can be calculated by eq.2, and the distance of a directional node transmits to a normal node can be calculated by eq.3.

$$
\begin{aligned}
L_{\text {od }} & =\sqrt[4]{G_{o} G_{o} \frac{h_{a}^{2}+h_{b}^{2}}{p_{r}}} \\
L_{d o} & =\sqrt[4]{G_{o} G_{m} \frac{h_{a}^{2}+h_{b}^{2}}{p_{r}}}
\end{aligned}
$$

When the receive threshold is same, we know $L_{\text {do }}$ larger than $L_{o d}$, so the directional node can reach farther distance. The communication distance of the two way of node is asymmetric.

We will describe the phenomenon more visible, as shown in Fig. 1, node A is equipped with smart antenna, and node B is equipped with Omni-directional antenna. In fig.1 (a), If node A has data to send to node B, A will transmit a directional RTS to node B, and B can receive properly, so only one hop reservation information is needed, while in Fig.1 (b), if node B has data to send to A, the Omni-directional RTS will not be heard by node A in Omni-directional model, B need to send data to the next hop of the routing table, until the data reach to A.

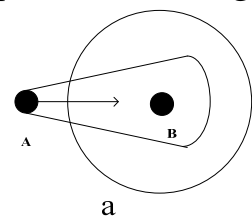

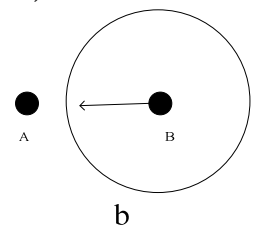

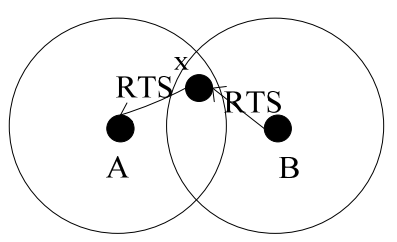

Figure 2 RTS forwarding

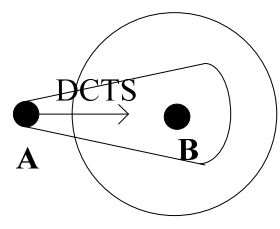

(1)

Generally, reducing communication hops can reduce the delay, and improve the channel utilization. Therefore, our research focuses on how to make the communication symmetric.

In wireless networks, the IEEE 802.11 DCF (Distributed coordinating function) often is used, DCF allows two kinds of work mode, if data packet is short enough, it will directly take DATA/ACK mode, or else it will use RTS/CTS/DATA/ACK mode for large data packets, because using VCS (Virtual Carrier Sense) can reduce conflicts, therefore, we use the second communication mode.

Like many DMAC protocols, we assume all nodes are in Omni-directional mode when the node is in idle state. This can effectively reduce the time of the channel reservation. Nodes that in the transmission range of normal node can receive properly, no matter what the antenna of a node uses, if the distance is larger than Omni-directional transmission range and smaller than the directional transmission range, the normal node will need more than one hop to send data packet. Therefore, if the transmitter is directional node, it will directly send to DRTS, if it is a normal node, it will send multi-hops to the receiver.

As shown in Fig.2, each node knows the information of its neighbors, including antenna type and location, if node $\mathrm{B}$ has data to send to the node $\mathrm{A}$, the node $\mathrm{A}$ is not a neighbor of $\mathrm{B}$ (O-O neighbors), because $\mathrm{A}$ is a directional nodes, the data communication between $\mathrm{B}$ and $\mathrm{A}$ can be finish in one hop, 
$\mathrm{B}$ can send RTS to $\mathrm{x}$, and $\mathrm{x}$ forward RTS to A, when A receives the RTS message, it will reply with directional CTS and know the channel reservation finish.

Two more address need to be added in the RTS frame, as shown in Table 1, in order to distinguish these new address from MAC address, the older address is called receiver and transmitter, the introduced address are called source (src) and destination (dst) which used to save the address of original communication. In addition, each node keeps an O-O routing table, which used to selecting the path from the source to the destination.

When MAC layer is received data from high layer, RTS will be send to reserve the channel. In our protocol, the node must judge which antenna it uses, if it is equipped with directional antenna, send the RTS packet directly to the receiver, in RTS frame, the receiver is the same as dst, the transmitter is the same as src, if the Node is it is equipped with Omni-directional antenna, it needs to execute the following change, the receiver will filled the next hops, the src field will filled with the destine. For example, the RTS frame from A to B and from B to A will be show in table 1.

Table 1 Example of RTS change

\begin{tabular}{|c|c|c|c|c|c|c|}
\hline \multirow{2}{*}{ direction } & \multicolumn{6}{|c|}{ MAC frame } \\
\cline { 2 - 7 } & $\ldots$ & receiver & transmitter & dst & src & $\ldots$ \\
\hline A->B & & B & A & B & A & \\
\hline B->A & & $\mathrm{x}$ & B & A & B & \\
\hline X->B & & A & $\mathrm{x}$ & A & B & \\
\hline
\end{tabular}

When a node receives the RTS packet, it is necessary to compare the destination with receiver fields, if it is equal, then reply a CTS to the source, if it is in the path of routing table, forward the RTS packet to the next hop, As shown in Fig.3, in order to complete the forwarding of the RTS, any intermediate node which receives the RTS message on the path does not set the NAV (Network Allocation Vector) table, only after received CTS,DATA and ACK will set the NAV / DNAV table.

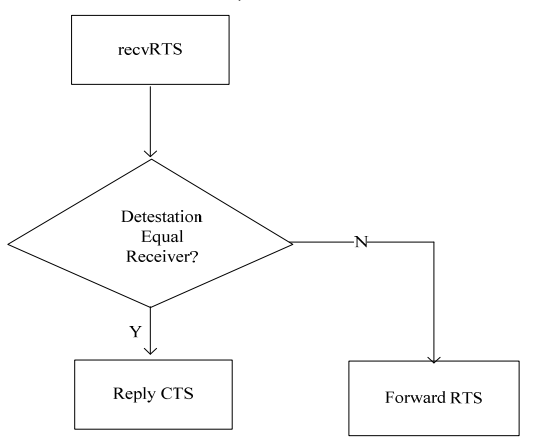

Figure 3 RecvRTS change

When the source receives the CTS packet, waiting for a SIFS time, and then starts transmitting data, after the destination receives DATA, directly sends an ACK packet to reply, a communication completed.

\section{Performance Analyses}

In order to verify the performance of the protocol, the simulation will be implementation in network simulator, 20 nodes are placed over a $150 * 150 \mathrm{~m}$ flat terrain. We use the static routing protocol NOAH. Ten directional nodes are used in this simulation, these nodes are Switched Beam Antenna and the beamwidths $60^{\circ}$, throughout and delay is always the measure of a network performance. We will compare our protocol with IEEE802_11DVCS [5] in throughput and delay.

According to Fig.4, when the data rate is small, both DFMAC and DVCS throughput are Linear increased, when the network load increases, using DFMAC protocol perform better than 802.11DVCS over $40 \%$.

As shown in Fig.5, when the data rate increases, the average delay of the DFMAC is smaller than 802.11DVCS protocol. DFMAC perform better. 


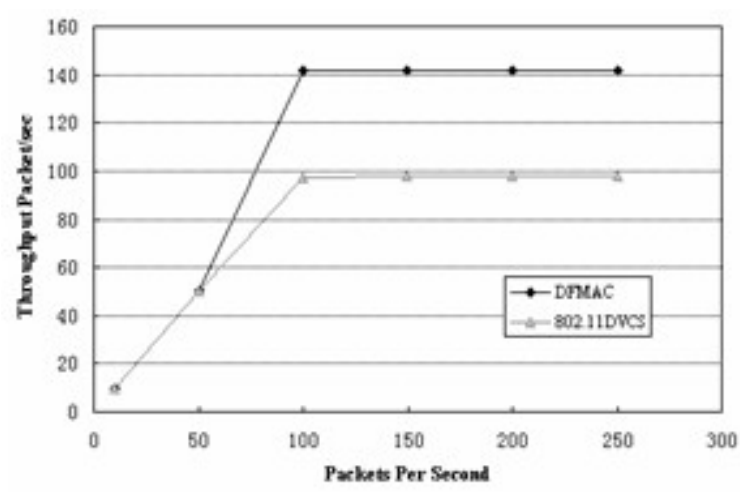

Figure 4 Average throughput

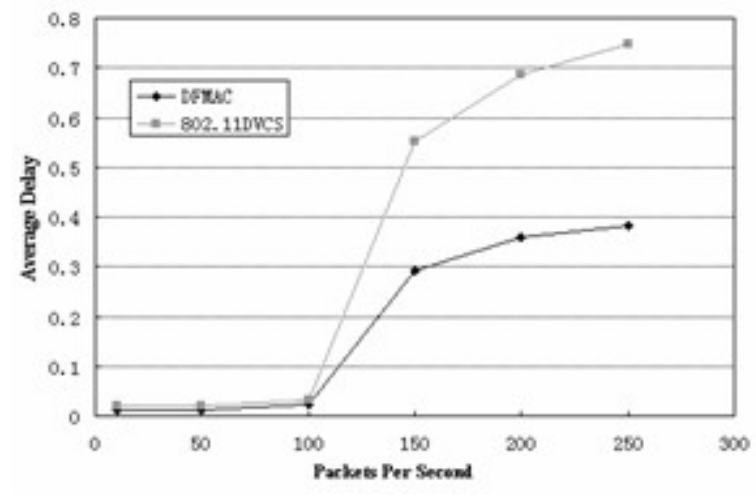

Figure 5 Average delay

\section{Conclusion}

There is few MAC protocol designed for hybrid network, especially for how to take the advantage of directional antenna, in this paper, we present the asymmetry problem of a hybrid network, and propose a DFMAC protocol, though using multi-RTS to solve this problem, the simulation results show that new MAC protocol can reduce communication time and improve the capacity of networks.

There may be some isolated nodes in wireless ad hoc between normal nodes and directional nodes. Our future research are will study how to solve isolated nodes without the assistance of other nodes.

\section{References}

[1] R. Ramanathan, J. Redi, C. Santivanez, D. Wiggins, and S. Polit. Ad hoc networking with directional antennas: A complete system solution [J]. IEEE Journal on Selected Areas in Communications, 2005, 23 (3):496.506.

[2] R. R. Choudhury and N. H.Vaidya. Deafness: a MAC problem in ad hoc networks when using directional antennas [C]. Proc of the 12th IEEE International Conference on Network Protocols, 2004:283-292.

[3] Zhang Jun, Jia Xiaohua. Capacity analysis of wireless mesh networks with omni or directional antennas [C]. Proc of IEEE INFOCOM, 2009:2881-2885.

[4] K. Sundaresan, R. Sivakumar. Cooperating with Smartness: Using Heterogeneous Smart Antennas in Ad-Hoc Networks [C]. Proc of IEEE INFOCOM, 2007:303-311.

[5] M. Takai, J. Martin, A. Ren R. Bagrodia. Directional Virtual Carrier Sensing for Directional Antennas in Mobile Ad Hoc Networks [C]. Proc of ACM MobiHoc, 2002: 39-46.

[6] Zhao Liqiang, Zhang Hailin, and Liu Yi. A MAC protocol to support hybrid antennas in wireless LAN [J]. JOURNAL OF ELECTRONICS 2007, 24 (3):309-315. 